\title{
BIBFRAME y el futuro de la descripción, vinculación y recuperación de los recursos de información
}

\author{
BIBFRAME and the future of the description, linking \\ and retrieval of information resources
}

Eder ÁVILA-BARRIENTOS 1 (D) 0000-0001-5439-0495

\begin{abstract}
Resumen
El propósito de este trabajo es analizar los avances de la implementación de Bibliographic Framework, BIBFRAME, en el ambiente de las bibliotecas, para identificar las tendencias futuras de la descripción, vinculación y recuperación de los recursos de información. Las complejas demandas de los usuarios de las bibliotecas han motivado la aparición de sofisticados métodos para la descripción de los nuevos recursos de información, los cuales proponen sugerentes cambios en la manera de recuperar dichos recursos, pues el modelo de datos vinculados en el que se fundamenta BIBFRAME pone de manifiesto el análisis de las relaciones que se establecen entre los datos de un determinado dominio de información documental. La sistematización de los datos de la biblioteca y su transformación en datos vinculados supone la generación de interfaces de búsqueda y recuperación altamente intuitivas y gráicamente atractivas para el usuario final. Así, el progreso de la implementación de BIBFRAME en los sistemas de información bibliotecarios dependerá de su capacidad y flexibilidad para estructurar los millones de registros Machine-Readable Cataloging que están disponibles en las bibliotecas a nivel mundial; además, se vislumbra el ambicioso objetivo de alcanzar la interoperabilidad universal entre los diferentes sistemas de información que están presentes en la Web.
\end{abstract}

Palabras clave: : BIBFRAME. Datos bibliográficos. Datos vinculados. MARC. Recursos de información.

\begin{abstract}
This work analyzes the implementation of BIBFRAME (Bibliographic Framework) in libraries, aiming to identify future trends in the description, linkage, and recovery of information resources. The complex demands of library users have led to the emergence of sophisticated methods for the description of new information resources. These methods propose suggestive changes in the recovery of such resources, since the model of linked data on which BIBFRAME is based reveals the analysis of the relationships between the data of a certain domain of documentary information. The systematization of the library's data and its transformation in linked data suppose the generation of search and recovery interfaces that are highly intuitive and graphically attractive for the final user. Thus, the progress of BIBFRAME implementation in library information systems will depend on its ability and flexibility to structure the millions of Machine-Readable Cataloging records that are available in libraries worldwide. In addition, we point to the ambitious goal of reaching universal interoperability, which is glimpsed among the different information systems that are present on the Web.
\end{abstract}

Keywords: BIBFRAME. Bibliographical data. Linked data. MARC. Information resources.

\footnotetext{
1 Universidad Nacional Autónoma de México, Coordinación de Humanidades, Instituto de Investigaciones Bibliotecológicas y de la Información. Circuito Interior, s/n., Torre II de Humanidades, Pisos 11, 12 y 13, Ciudad Universitaria, 04510, Distrito Federal, México. E-mail: <eder@iibi.unam.mx>.

Recibido el 20 de agosto de 2019, presentado el 5 de diciembre de 2019 y aprobado el 22 de abril de 2020.
}

Cómo citar este artículo/How to cite this article

Ávila-Barrientos, E. BIBFRAME y el futuro de la descripción, vinculación y recuperación de los recursos de información. Transinformação, v. 32, e190069, 2020. https://doi.org/10.1590/1678-9865202032e190069 


\section{Introducción}

Desde la publicación de los Functional Requeriments of Bibliographic Records, el cambio de paradigma de la catalogación de los recursos de información ha sido foco de atención de las comunidades bibliotecarias, pues ha motivado amplias reflexiones acerca de la evolución y adaptabilidad de los principios para la generación de registros bibliográficos y su conexión con fuentes externas al catálogo de las bibliotecas.

La aparición de "Resource, Description and Access (RDA)"en el 2010 (Joint ..., 2015), trajo consigo la necesidad de ejercer buenas prácticas en la descripción de los recursos de información. Además de propiciar un acceso eficiente a dichos recursos, pueden obtenerse datos consistentes para ser intercambiados, reutilizados y conectados en el ambiente Web.

Los registros bibliográficos y su disponibilidad en el catálogo en línea facilitan el acceso a los recursos de información que se encuentran en las colecciones de las bibliotecas, pues son un medio que responde a la demanda de los usuarios relativa a la recuperación y obtención de un recurso que responde a su demanda informativa. En este sentido, “La visión para el futuro [...] [en el marco de la catalogación] aún coloca al usuario en primer lugar, ya que proporciona la colocación y la diferenciación de entidades que son significativas para los usuarios" (Tillet, 2005, p. 205, destacado del autor).

Los recursos de información han evolucionado debido al impacto de las tecnologías digitales en sus estructuras. Para la comunidad bibliotecaria fue necesario replantear cuáles serían los elementos descriptivos que permitirían una eficiente organización de dichos recursos para fomentar su mejor recuperación y acceso por parte de los usuarios finales. Los principios tradicionales para la catalogación de los recursos se ven rebasados por las características que presentan los nuevos recursos de información, ya que la aparición de nuevos soportes y formatos para registrar la información, como es el caso de los audiovisuales, los conjuntos de datos y los documentos de origen digital, presentan atributos que tienen que representarse mediante nuevos elementos descriptivos. Además, los registros descriptivos resultantes deben integrarse en sistemas de información interoperables que permitan vincular los datos de la biblioteca. Por lo tanto, los catálogos en línea también deberán evolucionar y abrirse al ambiente Web.

Anteriormente, la información contenida en los recursos tenía que registrarse mediante principios universales, los cuales aún siguen vigentes. Taylor y Joudrey (2009), mediante un análisis efectuado a Hagler, manifiestan que la naturaleza de la organización de la información que ha sido registrada consiste en los siguientes procesos: (1) Identificar la existencia de todo tipo de recursos de información a medida que estén disponibles; (2) Identificar los trabajos contenidos en esos recursos de información o como parte de ellos; (3) Reunir sistemáticamente estos recursos de información en colecciones de bibliotecas, archivos, museos, archivos de Internet y otros depositarios similares; (4) Producir listas de estos recursos, preparadas de acuerdo con las normas estandarizadas; (5) Proporcionar nombre, título, tema y otro punto de acceso útil a estos recursos de información; (6) Proporcionar la localización de cada recurso de información o una copia de este.

Ronald Hagler (1997), en su libro "The Bibliographic Record and Information Technology", identificó estos procesos como parte de las seis funciones del control bibliográfico. Estas funciones han tenido una pronunciada influencia en la generación de nuevos modelos para la organización de la información.

De esta manera, para lograr la vinculación de los datos de las bibliotecas en el ambiente Web se requiere la compatibilidad del formato Machine-Readable Cataloging (MARC) con los principales estándares de los datos vinculados, por ejemplo su adaptación con Resource Description Framework (RDF) (y su codificación mediante Identificadores Uniformes de Recursos (URIs por su acrónimo en inglés), así como la viabilidad de su implementación en la creación de nuevas estructuras de datos y su latente incorporación en un nuevo sistema para el descubrimiento y localización de recursos. Estos aspectos han motivado la generación de estudios que analizan 
la viabilidad adaptativa de BIBFRAME. Xu, Hess y Akerman (2018, p. 245) han identificado que "los elementos MARC tienen asignaciones apropiadas para las declaraciones contenidas en BIBFRAME. Sin embargo, existen muchos cambios que son necesarios. Algunos de ellos se relacionan con la manera de asignar URIs a los datos MARC".

A su vez, Shieh (2018) ha expuesto los hallazgos que obtuvo el Programa de Catalogación Cooperativa de Library of Congress, respecto a la transición de los datos MARC a un entorno de datos vinculados. Como parte de este estudio se destacan las siguientes consideraciones: (a) La trasformación de los datos tendrá un impacto en su recuperación y descubrimiento; (b) Existen inconsistencias en la compatibilidad de los datos MARC con las declaraciones de BIBFRAME; (c) Los datos de la biblioteca deben alinearse con los principios de los datos vinculados, por ejemplo, con su flexibilidad para codificarse a manera de URIs.

La transformación de los datos localizados en los registros MARC Ileva implícita su estructuración, se deben eliminar puntuaciones y todos aquellos signos que puedan alterar la lectura y el procesamiento de los datos, ya que los registros de las bibliotecas contienen puntuaciones y signos que no son relevantes para la recuperación de los recursos de información. Por lo tanto, resulta pertinente plantear la modificación del registro catalográfico para la conformación de un registro descriptivo semántico con mayor flexibilidad para vincular datos.

Además, la utilización del vocabulario de BIBFRAME requiere de datos estructurados y consistentes que formen parte de una categoría en particular. Es decir, ¿los datos que se vincularán son bibliográficos, de autoridad o temáticos? ¿Forman parte de una colección especifica? ¿Responden a un tipo de recurso de información en particular? Algunos de los estudios que exponen la utilización y adaptación del vocabulario de BIBFRAME en diferentes tipos de datos correspondientes a recursos de información son los realizados por Tharani (2015), Balster, Rendall y Shrader (2018) y Taniguchi (2018).

Los datos deben describirse jerárquicamente para motivar su adaptación semántica mediante la aplicación de vocabularios y ontologías. Asimismo, no todos los datos que se encuentran en la biblioteca tendrán un vínculo con los recursos y contenidos del ambiente Web; en este aspecto recae la importancia de realizar buenas prácticas en la descripción de los recursos para fomentar la efectiva vinculación de los datos. "La nueva generación de sistemas integrados de bibliotecas tendrá en cuenta las sugerencias de los datos vinculados, proponiendo un nuevo modelo para la creación, el intercambio y la representación de datos" (Guerrini; Possemato, 2016, p. 188). Los nuevos sistemas de bibliotecas deberán ser interoperables y permitir la vinculación de los datos de la biblioteca con otros agentes y servicios disponibles en la Web.

Pues seguir con BIBFRAME es más prometedor que el estancamiento con MARC. Si diversas bibliotecas con diferentes necesidades bibliográficas trabajan juntas en el desarrollo continuo de BIBFRAME, puede ser lo suficientemente completo, versátil y robusto para asumir el mando que MARC ha mantenido durante casi medio siglo (Kroeger, 2013, p. 885).

De esta manera, el propósito de este documento es identificar los avances de la implementación de BIBFRAME en el ambiente de las bibliotecas para vislumbrar las tendencias futuras de la descripción, vinculación y recuperación de los recursos de información. Se presenta un análisis descriptivo de los proyectos y sistemas que exponen la implementación actual de BIBFRAME en el contexto de las bibliotecas con la finalidad de identificar las tendencias y el rumbo que la organización de la información puede tomar en un futuro inmediato.

\section{Datos vinculados y bibliotecas}

Los datos generados en las bibliotecas son principalmente de tres tipos: de autoridad, bibliográficos y temáticos. Estos datos se describen y asientan en los registros bibliográficos y de autoridad, los cuales están almacenados en las herramientas para la búsqueda, recuperación y acceso a la información; por ejemplo, en catálogos en línea, repositorios, bases de datos y descubridores de información. 
El intercambio de registros ha permitido la conformación de catálogos en línea cooperativos, los cuales hacen referencia a recursos de información de una amplia variedad temática y tipología. Los datos que conforman estos recursos bien pueden conectarse con otras fuentes externas al catálogo mediante un proceso interoperable fundamentado en la aplicación de los principios de los datos vinculados (Linked Data).

Los datos vinculados son estructuras de datos que tienen el potencial de vincularse con otros datos que están disponibles en el ambiente de la Web, mediante un proceso de interoperabilidad y buenas prácticas. BernersLee (2006) definió cuatro principios para construir datos vinculados en el ambiente Web: (1) Utilizar URIs como nombres para las cosas; (2) Utilizar el protocolo HTTP para que las personas puedan buscar esos nombres; (3) Utilizar RDF y SPARQL para enriquecer esos URIs y proporcionar información adicional; (4) Incluir enlaces a otros URIs para descubrir más cosas.

Cuando los principios de los datos vinculados son aplicados en los datos de la biblioteca, es posible generar esquemas y vocabularios para la representación de los datos y su latente vinculación en el ambiente Web. "Al utilizar identificadores uniformes de recursos (URI's) para designar obras, lugares, personas, eventos, temas y otros objetos o conceptos de interés, las bibliotecas permitirán que los recursos se citen en una amplia gama de fuentes de datos" (W3C, 2011).

De esta manera, la reutilización de los datos de la biblioteca en el ambiente Web es parte de un proceso interoperable, mediante el cual se vinculan los datos bibliotecarios con otras fuentes de datos, siempre y cuando exista un vínculo de significado o similitud entre sus atributos.

Aunado a ello, los catálogos en línea de las bibliotecas deberán integrarse con otras herramientas de descubrimiento y permitir la vinculación de los datos que se encuentran almacenados y representados en sus registros bibliográficos y de autoridad. En los últimos años han aparecido diversos desarrollos que tienen el propósito de convertir los datos de la biblioteca en estructuras compatibles con los principios de Linked. Data, para fomentar su vinculación en el entorno de la Web. The British Library Metadata Servicies (British ..., 2020) es uno de los desarrollos que pone de manifiesto la conversión de diversas colecciones de datos en formatos RDF/XML y N-Triples.

A su vez, han surgido un amplio número de lenguajes, vocabularios y ontologías con la intención de construir estructuras interoperables que fomenten la integración de los datos de las bibliotecas mediante el uso de agregadores semánticos. Linked Open Vocabularies y Open Metadata Registry son dos plataformas que ejemplifican a estos tipos de elementos.

\section{BIBFRAME}

"Bibliographic Framework (BIBFRAME) es una iniciativa que pretende evolucionar los estándares de descripción bibliográfica a un modelo de datos vinculados, con el fin de que la información bibliográfica sea más útil tanto dentro como fuera de la comunidad bibliotecaria" (Library of Congress, 2016).

Los datos de las bibliotecas son de gran valor para el desarrollo y estructuración de la Web semántica, han pasado por una serie de procesos analíticos y normativos que les confieren un grado mayor de confiabilidad y calidad.

El modelo 2.0 de BIBFRAME plantea organizar los datos de la biblioteca en tres niveles de abstracción: obra, instancia (manifestación, expresión) y ejemplar. Además, el modelo utiliza tres conceptos clave para el establecimiento de relaciones entre los datos: agente (que pueden ser personas, organizaciones y jurisdicciones), tema y evento. 
El modelo de BIBFRAME se encuentra ampliamente influenciado por RDA, pues son dos iniciativas que además de pretender la descripción efectiva de los recursos, fomentan el intercambio de los datos y su latente vinculación con fuentes externas al catálogo en línea de la biblioteca.

El vocabulario de BIBFRAME está conformado por clases y propiedades RDF. Las tres clases principales son obra, instancias y ejemplares. Además, el vocabulario incluye subclases que se relacionan directamente con clases las principales. Por otra parte, las propiedades del vocabulario describen las características de los recursos y las relaciones que pueden establecerse entre ellos (Figura 1).

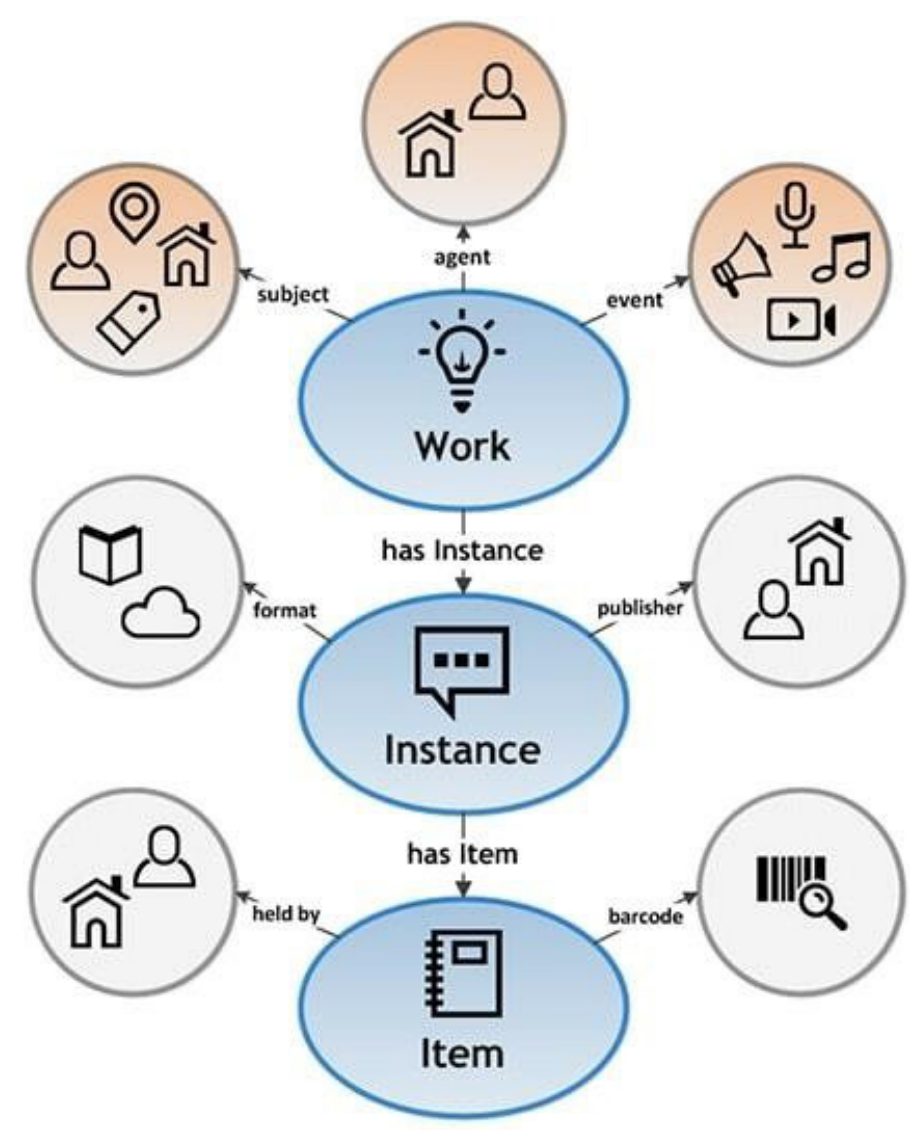

Figura 1. BIBFRAME Model 2.0.

Fuente: Library of Congress (2016).

Las relaciones entre los datos deben explicar el significado que existe entre la unión de dos o varios recursos que sean derivados o reciban influencia de una determinada obra. Es en este aspecto que recae la relevancia de impulsar la generación de nuevos modelos para la descripción, vinculación y recuperación de los recursos. El análisis de las similitudes existentes entre los datos que conforman dichos recursos puede explicarse mediante el establecimiento de vinculaciones de significado. Se trata de explotar el potencial de los datos de la biblioteca para descubrir los recursos y contenidos que se vinculan con una demanda de información en particular. 
BIBFRAME ha sido desarrollado con una mirada cuidadosa en los diversos modelos emergentes de la comunidad bibliográfica, ajustándose a la visión actual del mundo de Internet utilizando "reglas" de datos vinculados. Pero algunas de las prácticas comunes de los datos bibliográficos no coinciden con la visión de los datos vinculados (McCallum, 2017, p. 81).

Por lo tanto, la implementación de BIBFRAME en las bibliotecas dependerá de un proceso de adaptación en el cual intervienen principios que involucran la óptima integración y adaptabilidad de los datos de la biblioteca con otros sistemas de información ajenos a su estructura.

Se trata de motivar la generación de un modelo para la descripción de recursos de información que sea compatible con la Web, para propiciar la vinculación de estos recursos y los contenidos que forman parte de sus estructuras. De esta manera, las bibliotecas pueden participar en otros ámbitos de acción en donde la información es parte fundamental para la toma de decisiones y en donde el intercambio y generación de nuevos conocimientos es una constante.

\section{Desarrollos actuales}

El progreso actual de la implementación de BIBFRAME se resume en dos ejes principales: por un lado, la generación de proyectos que integran los datos de la biblioteca y su estructuración mediante BIBFRAME y, por el otro, la conformación de aplicaciones informáticas con una arquitectura configurada acorde a los principios de los datos vinculados para su integración con los actuales sistemas de las bibliotecas.

En el Cuadro 1 se exponen y describen los proyectos que se relacionan con la implementación de BIBFRAME. Puede observarse que en dichos proyectos se toman en cuenta los datos correspondientes a diferentes tipos de recursos de información. Además, se incluye el caso del software Zepheira (2020), pues en diversos estudios de caso

Cuadro 1. Proyectos y desarrollos informáticos relacionados con la implementación de BIBFRAME.

\begin{tabular}{ll}
\hline Nombre de proyecto & Descripción \\
\hline Share Virtual Discovery Environment & Prototipo que tiene el objetivo de establecer un entorno para el uso de datos vinculados de bibliotecas \\
dentro de un contexto global. Ofrece una interfaz para la búsqueda y acceso a recursos bibliográficos & de las bibliotecas de las siguientes instituciones académicas: Stanford University, University of California \\
& Berkeley, Yale University, Library of Congress, University of Chicago, University of Michigan Ann Arbor, \\
& Harvard University, Massachusetts Institute of Technology, Cornell University, Columbia University, University \\
& of Pennsylvania, Texas A\&M University y University of Toronto.
\end{tabular}

Share Catalogue Plataforma que permite navegar por catálogos en línea bibliográficos organizados de acuerdo con el modelo BIBFRAME. Es un proyecto italiano en el cual participan las siguientes universidades: Università degli Studi di Napoli Federico II, Università degli Studi della Basilicata, Università degli Studi del Sannio, Università degli Studi di Salerno, Università degli Studi di Napoli Parthenope, Università degli Studi del Salento, Università degli Studi di Napoli L'Orientale, Università degli studi della Campania Luigi Vanvitelli.

Bibframe 2.0 Digitized Books. University Library, Proyecto centrado en la colección digital de las novelas inglesas del siglo XIX disponibles en la University of Illinois at Champaign Urbana Biblioteca Urbana-Champaign de la Universidad de Illinois. Se transformaron 7,829 registros de Dublin Core a BIBFRAME 2.0, y se desarrolló una interfaz para su descubrimiento.

Zepheira Software propietario que, mediante diversas aplicaciones informáticas, tiene el propósito de transformar el catálogo de la biblioteca, mediante vocabularios de datos vinculados, incluyendo en modelo 2.0 de BIBFRAME.

@Cult. LOD Plattform Herramienta informática para estructurar catálogos en línea bibliográficos, organizados de acuerdo con el modelo de datos BIBFRAME para su posterior publicación como datos abiertos vinculados Linked Open Data.

Fuente: Elaborado por el autor (2019). 
y pruebas piloto que se han examinado, se menciona su ejecución como parte del procesamiento de los datos bibliográficos y su estructuración mediante los principios de los datos vinculados. Zepheira ha sido el software elegido por Library of Congress para comenzar con los trabajos de la implementación de BIBFRAME en diferentes colecciones de recursos de información.

La descripción de los recursos mediante BIBFRAME contempla una transición normativa y pragmática de los procesos de organización de la información. Es decir, las bibliotecas deben generar propuestas innovadoras que propicien el acceso universal a los recursos, de una manera sistematizada, intuitiva e integradora para el usuario final, esto supone alcanzar una flexibilidad en sus principios organizativos.

Los actores de las bibliotecas deben contar con una visión integradora al cambio, pues en la actualidad las complejas demandas informativas del usuario y el contexto datificado en donde interactúa, hacen necesario contar con nuevos modelos y pautas para la descripción de los recursos. Para ello, es necesario comprender los cambios que se avecinan y de qué manera las comunidades bibliotecarias pueden enfrentarlos y adaptarlos en sus respectivos contextos.

En la Figura 2 puede apreciarse un ejemplo de la descripción efectuada al libro impreso de "Ulises" de James Joyce. Esta descripción ha generado una visualización en forma de grafo de la instancia del recurso. El reto principal de este tipo de visualización consiste en ofrecer un método de acceso y recuperación visualmente atractivo e intuitivo para el usuario final.

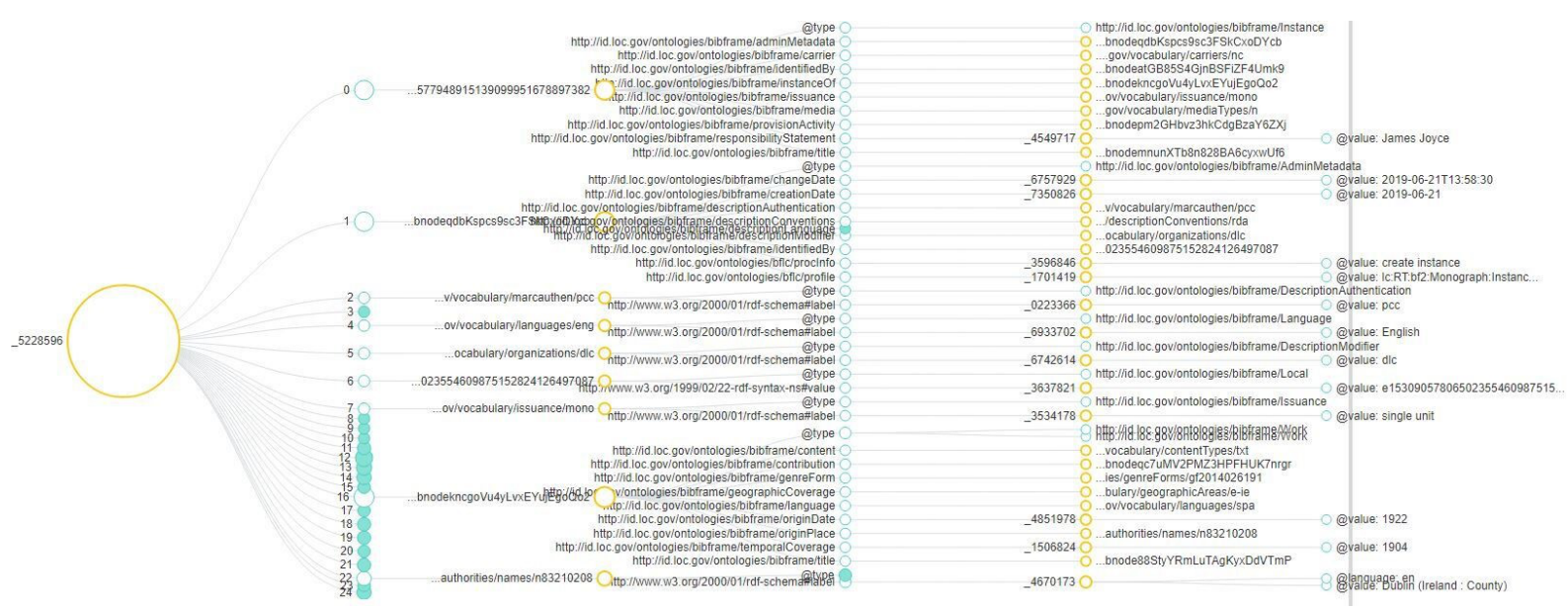

Figura 2. Visualización en modalidad de grafo de la instancia Ulises de James Joyce, desarrollada mediante BIBFRAME Editor. Fuente: Elaborado por el autor (2019).

La interacción del usuario con los datos vinculados que se generen en el marco de las bibliotecas deberá contemplar la formulación de nuevos métodos para recuperar la información. El modelo de datos vinculados contempla su usabilidad en concordancia con el significado del contexto en donde se localicen y la demanda informativa que el usuario manifieste al momento de recuperarlos.

Por otra parte, BIBFRAME tiene alcances y limitaciones que conviene tener presentes. Taniguchi pone en cuestión su implementación como un esquema adecuado para intercambiar y compartir metadatos descriptivos sobre diversos recursos bibliográficos y afirma que: 
La política de restricción de dominio de BIBFRAME puede no ser adecuada para intercambiar y compartir esquemas de metadatos descriptivos sobre diversos recursos bibliográficos. El método utilizado por BIBFRAME debe ser reconsiderado. Además, sería posible examinar BIBFRAME desde diferentes puntos de vista relacionados con el intercambio y compartición de esquemas de metadatos. Dicha investigación es necesaria para juzgar la idoneidad de BIBFRAME en la actualidad (Taniguchi, 2018, p. 60).

Bajo esta premisa, el avance en la implementación de BIBFRAME en el ambiente de las bibliotecas será significativo para ejercer descripciones semánticas de los recursos de información que son publicados de manera impresa pero también aquellos que nacen como parte de procesos de creación digital. La intención principal consiste en vincular datos que estén correctamente representados en esquemas de metadatos flexibles e interoperables.

La descripción semántica de un recurso de información consiste en registrar sus atributos en forma de datos estructurados y explicar las vinculaciones que estos puedan tener con otras fuentes disponibles en la Web. Se trata de una descripción integradora que se fundamenta en la aplicación de principios normativos de índole bibliotecológica y tecnológica. Se estima que la integración de ambos principios fomentará la generación de descripciones altamente enriquecedoras.

La aplicación de BIBFRAME en las actividades de organización de la información pone de manifiesto la aparición de nuevos procesos para fomentar el acceso universal a los recursos mediante la explotación de los datos que los caracterizan. Los datos disponibles en los registros MARC tendrán que extender su alcance más allá de representar los atributos del recurso y de fomentar la localización y búsqueda de información en la biblioteca.

En el contexto actual, es necesario que los datos generados en las bibliotecas se conecten con otros escenarios que permitan al usuario incrementar sus opciones para atender sus demandas informativas, ya que un dato en particular que se encuentre disponible en el catálogo de la biblioteca puede propiciar la recuperación de múltiples recursos y contenidos con atributos similares y que estén disponibles en el ambiente digital. Para ello, será pertinente realizar registros descriptivos que fomenten la vinculación de los datos mediante el ejercicio de buenas prácticas en el ámbito de la descripción semántica de los recursos.

Es hora de que las bibliotecas se unan al movimiento de los datos vinculados en números más grandes. BIBFRAME no es el único proyecto de datos vinculados, pero dado que está siendo desarrollado por la Biblioteca del Congreso, lo más probable es que represente una respuesta popular para la adaptación de los datos vinculados (Steele, 2018, p. 11).

Esta adaptación requerirá que las bibliotecas encuentren un proceso unificador que integre los diversos modelos normativos que utilizan para la organización de los recursos de información. La descripción de estos recursos tendrá impacto en su búsqueda, recuperación y acceso de manera integradora.

Las bibliotecas tienen que ofrecer innovadoras alternativas para la organización de los recursos de información y actualizar sus principios organizativos. En la actualidad, los sofisticados sistemas de información disponibles en la Web ejercen búsquedas de información con mayor integridad, lo cual ha captado la atención de los usuarios que buscan satisfacer su demanda informativa de manera casi instantánea de una forma remota, poniendo de manifiesto nuevos comportamientos en la búsqueda y recuperación de información.

Anteriormente, Eliot Christian tuvo la peculiar idea de generar un servicio Global de Localización de Información, el cual fue diseñado para que las personas encontraran y recuperaran información fácilmente, incluso cuando las fuentes de información se expandieran y diversificaran. En este sistema, los catálogos en línea de las bibliotecas fungirían como proveedores e intermediarios de información mediante una comunicación interoperable entre los sistemas disponibles en la Web y los catálogos en línea de las bibliotecas. En este sentido, "la interoperabilidad describe la capacidad que tienen los diferentes sistemas, productos o proveedores para interactuar entre sí. Los sistemas perfectamente interoperables pueden intercambiar e interpretar información con 
total libertad" (Kalso, 2019). La idea de disponer de un sistema de información con interoperabilidad universal no es reciente, con la aparición de novedosos métodos para la vinculación de los datos en el ambiente de las bibliotecas y su conexión con la Web; esta idea progresa paulatinamente en función de la aparición de nuevos desarrollos normativos.

A su vez, se tienen que considerar los aspectos técnicos, legales y económicos que intervienen en la implementación de BIBFRAME en las bibliotecas. Estos aspectos llegan a convertirse en barreras para la reutilización y el libre acceso a los datos que forman parte de los recursos de información, pues la información también forma parte de un contexto globalizado y capitalizado. Las comunidades bibliotecarias y otras instituciones culturales deberán trabajar juntas para desarrollar buenas prácticas en la construcción de datos vinculados.

Los datos vinculados se han convertido en una palabra de moda en el control bibliográfico de la biblioteca y en los servicios de descubrimiento debido a las oportunidades y posibilidades que representan para las bibliotecas (verificar esta alteración, pues se trata de una cita). Sin embargo, hay muchos desafíos que debemos enfrentar como comunidad (Cole et al., 2013, p. 191).

Por lo tanto, la adaptación de BIBFRAME en el contexto de las bibliotecas deberá fundamentarse en un cambio estructural que fomente el desarrollo de buenas e innovadoras prácticas para la organización de los recursos de información. La biblioteca debe adaptarse al cambio tecnológico provocado por el contexto datificado que la rodea.

\section{Consideraciones Finales y Tendencias Futuras}

El propósito principal de BIBFRAME es generar un modelo para la descripción de los recursos fundamentado en la lógica estructural de los datos vinculados, también contempla su implementación en nuevos sistemas, un aspecto que permite definir la tendencia futura de la evolución del catálogo en línea de la biblioteca. Además, la actualización de los sistemas integrales de las bibliotecas pone de manifiesto la incorporación de nuevos modelos, formatos y esquemas para la descripción de los recursos.

Para que esta actualización tenga un efecto esperanzador se necesita la colaboración de los diversos actores que forman parte de los procesos de organización de la información en las bibliotecas, pues BIBFRAME marcará un cambio de paradigma en la manera de organizar los recursos de información.

El catálogo en línea de la biblioteca deberá comunicarse con los diversos sistemas de información disponibles en la Web, mediante un proceso de interoperabilidad. De esta manera, los registros MARC podrán vincularse con otros datos disponibles en el ciberespacio. Además, el formato MARC deberá flexibilizarse y adaptarse a los principios de los datos vinculados; BIBFRAME es una propuesta idónea para alcanzar este nivel de adaptabilidad.

Los catálogos en línea de las bibliotecas deberán ofrecer métodos de recuperación más eficientes, caracterizados por la interacción intuitiva, gráfica e integradora por parte del usuario, pues la consulta y acceso a los resultados de búsqueda mediante la estructura de los datos vinculados contempla la implementación de interfaces que hagan posible el acceso a los datos y sus respectivas vinculaciones.

La eventual implementación de BIBFRAME en los sistemas de información bibliotecarios, dependerá de su capacidad y flexibilidad para adaptar a la abismal cantidad de datos codificados en MARC que están disponibles en sus catálogos en línea.

El futuro de la organización de la información se encuentra en el desarrollo de innovadores métodos para sistematizar, organizar y recuperar los datos disponibles en las bibliotecas. Será imprescindible que estas propuestas beneficien a los usuarios en la satisfacción de sus demandas informativas, además de propiciar la interacción de los datos de la biblioteca en el complejo y progresivo ciberespacio. 


\section{Referencias}

Balster, K.; Rendall, R.; Shrader, T. Linked serial data: mapping the CONSER standard record to BIBFRAME. Cataloging \& Classification Quarterly, v. 56, n. 2/3, p. 251-261. Doi: https:// doi.org/10.1080/01639374.2017.1364316.

Berners-Lee, T. Linked Data. W3C, Cambridge, 2006. Available from: https://www.w3.org/Designlssues/LinkedData.html. Access: June 18, 2019.

British Library. Collection metadata: metadata services. London: British Library, 2020. Available from: http://www.bl.uk/ bibliographic/datafree.html. Access: June 18, 2019.

Cole, T. et al. Library MARC records into linked open data: challenges and opportunities. Journal of Library Metadata, $\mathrm{v}$. 13, n. 2/3, p. 163-196, 2013. Doi: https://doi.org/10.1080/193 86389.2013.826074.

Guerrini, M.; Possemato, T. From record management to data management: RDA and new application models BIBFRAME, RIMMF, and Olisuite/WeCat. Cataloging \& Classification Quarterly, v. 54, n. 3, p. 179-199, 2016. Doi: https://doi.org/10.1 080/01639374.2016.1144667.

Hagler, R. The bibliographic record and information technology. Chicago: American Library Association, 1997.

Joint Steering Committee for Development of RDA. RDA: Resource Description and Acces USA: JSC, 2015. Available from: http://www.rda-jsc.org/archivedsite/rda.html\#background. Access: June 15, 2019.

Kalso, R. Interoperability. Hackensack: Salem Press, 2019. Available from: http://search.ebscohost.com/login.aspx?dir

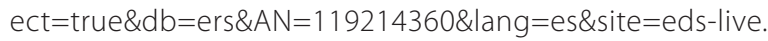
Access: June 17, 2019.

Kroeger, A. The road to BIBFRAME: the evolution of the idea of bibliographic transition into a post-MARC future. Cataloging \& Classification Quarterly, v. 51, n. 8, p. 873-890, 2013. Doi: https://doi.org/10.1080/01639374.2013.823584.
Library Linked Data Incubator Group final report. W3C, Chicago, 2011. Available from: http://www.w3.org/2005/ Incubator/IId/XGR-IId-20111025/. Access: June 18, 2019.

Library of Congress. Overview of the BIBFRAME 2.0 Model. Library of Congress, Washington, 2016. Disponible en: https:// www.loc.gov/bibframe/docs/bibframe2-model.html. Access: June 18, 2019.

McCallum, S. BIBFRAME Development. Italian Journal of Library, Archives, and Information Science, v. 8, n. 5, p. 71-85, 2017. Doi: https://doi.org/10.4403/jlis.it-12415.

Shieh, J. Reports from the program for cooperative cataloging task groups on URIs in MARC \& BIBFRAME. Italian Journal of Library, Archives, and Information Science, v. 9, n. 1, p. 110-119, 2018. Doi: https://doi.org/10.4403/jlis.it-12052.

Steele, T. What comes next: understanding BIBFRAME. Library Hi Tech, v. 33, n. 3, p. 1-13, 2018. Doi: https://doi.org/10.1108/ LHT-06-2018-0085.

Taniguchi, S. Is BIBFRAME 2.0 a suitable schema for exchanging and sharing diverse descriptive metadata about bibliographic resources? Cataloging \& Classification Quarterly, v. 56, n. 1, p. 40-61, 2018. Doi: https://doi.org/10.1080/01639374.2017.1382643.

Taylor, A.; Joudrey, D. The organization of information. Westport: Libraries Unlimited, 2009.

Tharani, K. Linked Data in libraries: a case study of harvesting and sharing bibliographic metadata with BIBFRAME. Technology and Libraries, v. 34, n. 1, p. 5-19, 2015. Doi: https:// doi.org/10.6017/ital.v34i1.5664.

Tillet, B. FRBR and cataloging for the future. Cataloging \& Classification Quarterly, v. 39, n. 3/4, p. 197-205, 2005. Doi: https://doi.org/10.1300/J104v39n03_12.

Xu, A.; Hess, K.; Akerman, L. From MARC to BIBFRAME 2.0. Crosswalks. Cataloging \& Classification Quarterly, v. 56, n. 2/3, p. 224-250, 2018. Doi: https:/doi.org/10.1080/01639374.2017. 1388326 .

Zepheira. Zepheira: Linked Data. Ipswich: Ebsco, 2020. Available from: https://zepheira.com/. Access: May 15, 2020. 